International Journal of Engineering \& Technology, $7(2.24)(2018) 255-258$
International Journal of Engineering \& Technology
SPC
Website: www.sciencepubco.com/index.php/IJET
Research paper

\title{
Mobility Platform for Physically Challenged People using Wireless Sensor Network
}

\author{
K. $\operatorname{Vidya}^{1^{*}}$, A. Aarthy ${ }^{2}$ \\ Veltech Multitech Engineering College, Avadi, Chennai, TamilNadu, India \\ *Corresponding Author E-mail: vidya@veltechmultitech.org
}

\begin{abstract}
The Primary objective of Automatic Railway Bridge System is to help the physically Challenged Passenger to move from one Platform to another. Crossing the railway track inside the railway station is very difficult. But it is quite difficult to the handicapped and aged persons to cross the railway track without the help of other. The proposed system uses the train time for opening and closing of bridges. This is done by wireless communication. Each train is connected with RF transceiver, which will send a data of arrival before it reaches the station platform. If the train leaves the platform, the automatically the mobile platform will be bridged between two platforms to build a path between them. This project is designed with arduino microcontroller. The arduino microcontroller gives the input from the RSSI and give command to the motor to run in order to change in the position of open and close the mobility rostrum
\end{abstract}

Keywords: Rostrum, Arduino, RSSI, Platform

\section{Introduction}

Indian railway network is the one of the biggest rail network in Asia. To make it a safe and reliable system is an enormous challenge. Unmanageable platform crossings are one of the problem areas for the Indian Railways, and one of the major issues of death. In spite of various measures taken by the Indian Railways, platform crossing deaths have continued to occur, that too frequently. Crossing the railway track inside the railway station is very tedious especially for handicapped and disabled person. They find it quite difficult to walk over the over bridge for crossing the platform.

To solve this problem we use a new approach called Automatic Railway Bridge System. Recently, advancements in information technology have enabled applying sensors to railway environments such as PIR sensors. Also technology for wireless data transfer has also been developed which helps in updating the train timing. The objective of PIR sensor in railway station is to sense passenger's movements on the bridge before it opens. Therefore when emergency situation arises, immediate recognition and response to the situation is possible.

In this project, we not only ensure an easy method for track crossing but also platform safety. It shows train signal (red or green) and if necessary we can use audio telling the arrival of train before stepping over the bridge. If necessary, for safety opening and closing of bridge we can use train navigation system, RUNE system. This project introduces overall system overview, configuration and detection process of the system.

The mobility platform are connected in the centre of the railway tracks in the side of the Platforms. At the time when there is no train arrival in the station the desired Platforms will be opened. The physically challenged people will use the moving Platform from one platform to another Platforms. Whenever train is arriving, moving Platforms will be close.
Whenever train entry will be declared in a voice announcement and LED signal will display. The existing system of fundamental disadvantage is Indian railway is moving up on the fly overs for a physically challenged people are inconvenient to cross the bridge. The proposed system gives a superior answer for exchanging physically challenged people in one Platform to another Platform without utilizing fly overs. The mobile plates between two Platforms associate the Platforms and the lift consequently moves.

\section{Hardware Setup}

The proposed system has remote association from transmission unit to getting unit. The Mobile Platforms moves like a lift which is more point of preference than existing system. In mobility platform system, whenever the train near to arrives the platform, the mobility rostrum automatically will open by intimating a warning like buzzer will be ringing, signal light change into green to red and also display a warning "PLEASE DO NOT USE THE MOBILITY PLATFORM ROSTRUM" to the passengers those who are using the mobility platform. This is done by wireless communication. The LED signal sign is utilized to show the train arriving and departure in the railway station. The remote connection from transmitter to beneficiary lessen the establishment hardware of the framework. In the controller unit the voice declaration, LED sign and engine units are controlled. 


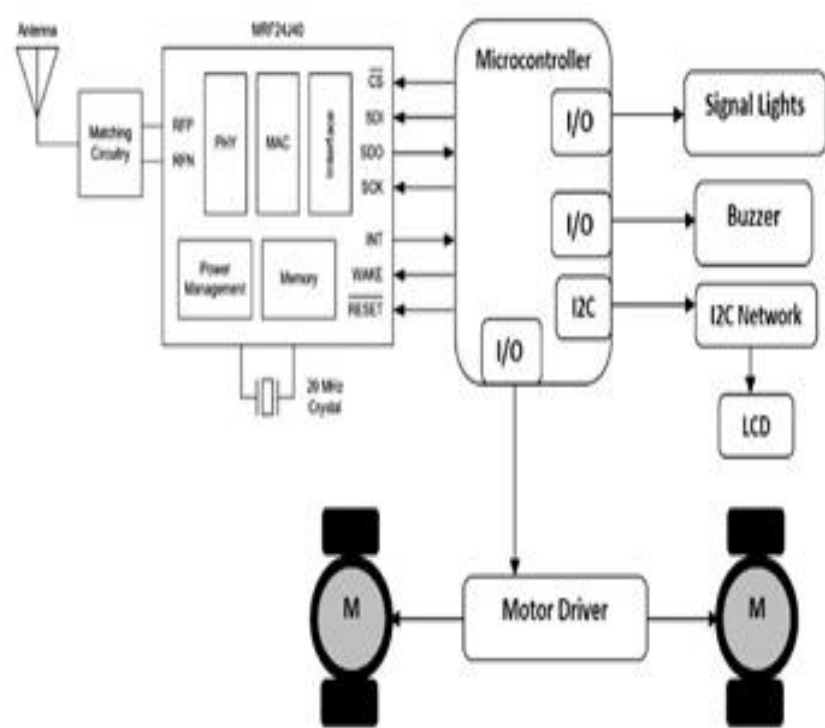

Fig. 1: Block Diagram of Mobility Platform

In the mobile platform unit, it moves to associate the intersection Platforms and begin to move the lift. The physically challenged people will utilize the Mobile Platform to cross the intersection Platforms. Show in fig: (1)\&(2)

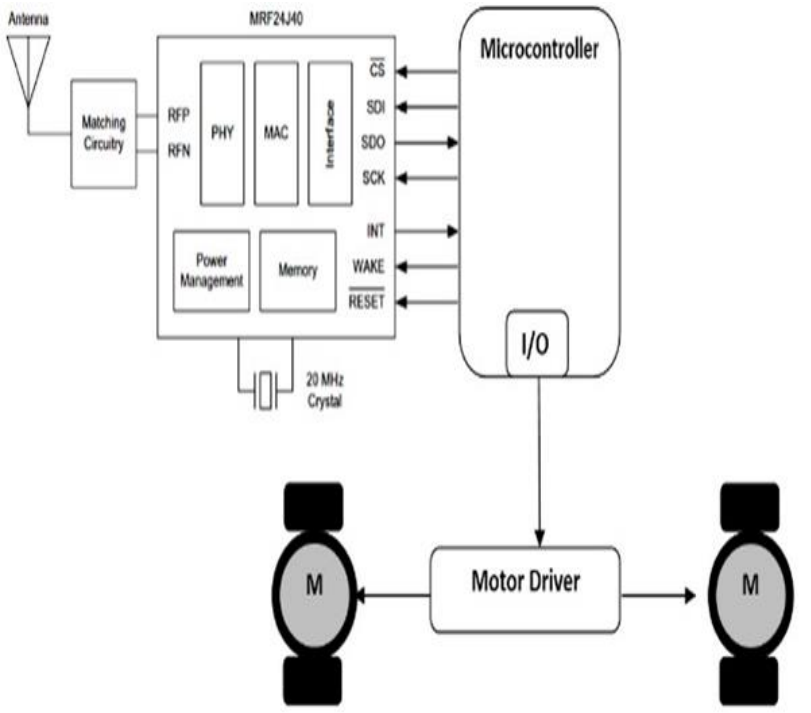

Fig. 2: Block Diagram of Train

\section{System Design}

The platform design shown in figure $1 \& 2$ for moving platform is described in this paper. The microchip (MRF24J40) is used to transmit \& receive the signal of RF transmitter and receiver, then it sent the information to the microcontroller. Microcontroller is used to control by giving inputs to the signal light, buzzer, i2c network through LCD and motor driver. Signal light is used to indicate the people. When green light is indicate the people use the moving platform and red light indicate the train arrival. Buzzer is also used to indicate the people.I2c is used to collect the date and time. LCD is used to display the RSSI value of the train distance. Mobility platform done using Radio Signal Strength Indicator (RSSI) under WPAN communication system, where radio frequency transmitted and receiving through transceiver in WPAN communication system

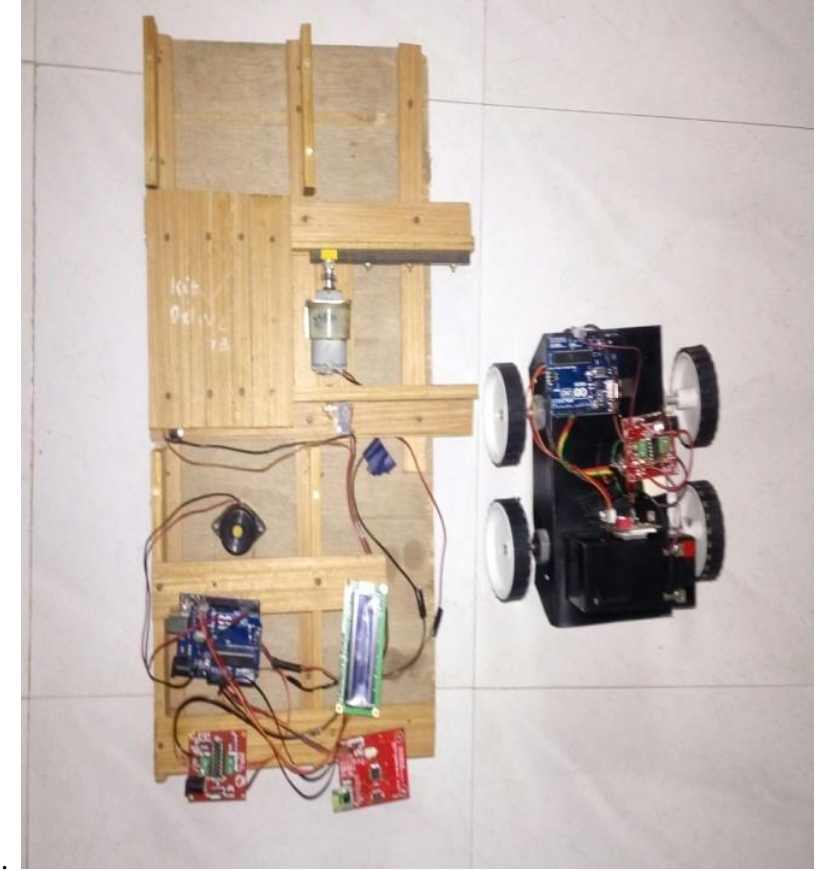

Fig. 3: Hardware Assembly of normal setup

\section{Working of the System}

\section{RSSI}

RSSI (Received Signal Strength Indicator) is used to measure the distance of train. The Sensor (RF transmitter and receiver) is used to sense the arrival of the train and also sense the dispatcher. The sensor signal is connect to the microcontroller (arduino) show the RSSI range value of the train distance. RSSI is wireless networking of IEEE 802.11 protocol family which is basically used for measurement of the received RF power currently.

\section{MRF24J40 MICROCHIP}

Power supply to the microcontroller which is use in the system as the capable of storing the data by using the MRF24J40 microchip. The protocol provides reliable direct wireless communication via an easy-to-use programming interface.

The microchip (MRF24J40) is used to transmit and receive the signal of RF transmitter and receiver, then it send the information to the microcontroller.

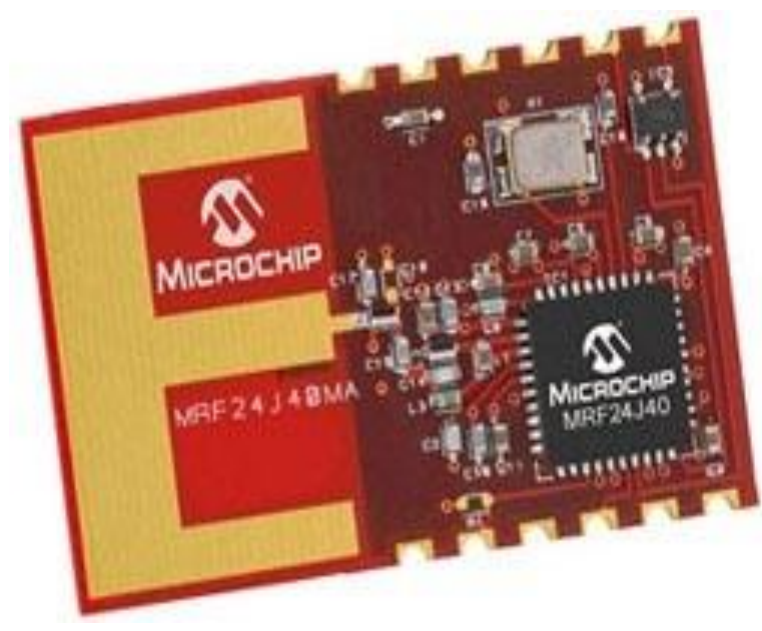

Fig. 3: $\mathrm{MiWi}$ 


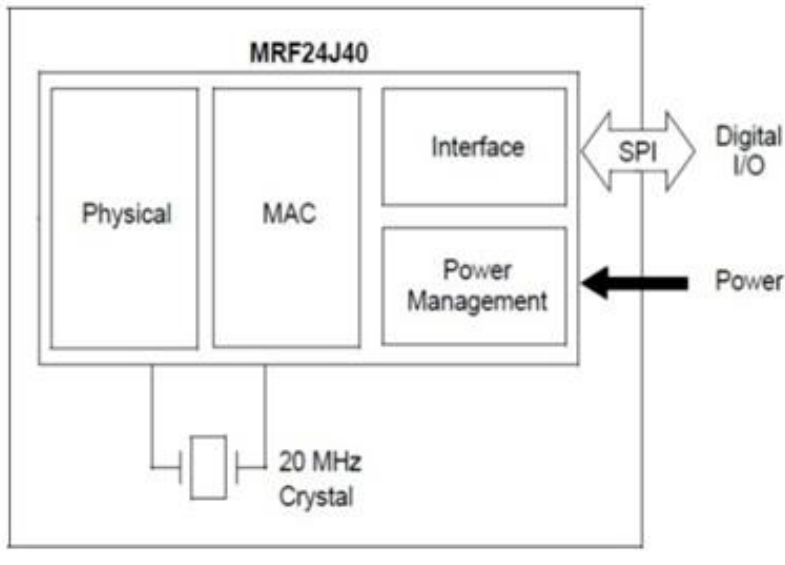

Fig. 4: MRF24J40 Microchip

\section{ARDUINO MICROCONTROLLER}

This microcontroller is used in this project to control and giving inputs to the signal light, buzzer, i2c network through LCD and motor drive. The microcontroller support needed everything; simply connect it with a AC-to-DC adapter or battery to get started.

\section{LCD}

LCD is used to display the RSSI value. If the RSSI value below 41 the LCD display show the green signal so, that the people can move to the one platform to another platform. If the range value is exceed 41 the LCD display indicate the red signal so, that it indicate the arrival of the train.

\section{Result}

In this section the both simulation and hardware results are discussed.

\section{1) Simulation Result}

The simulation design is developed in PROTEUS software. The coding is done by using embedded c language. In this also there is a simulation result for both arrival and dispatcher train.

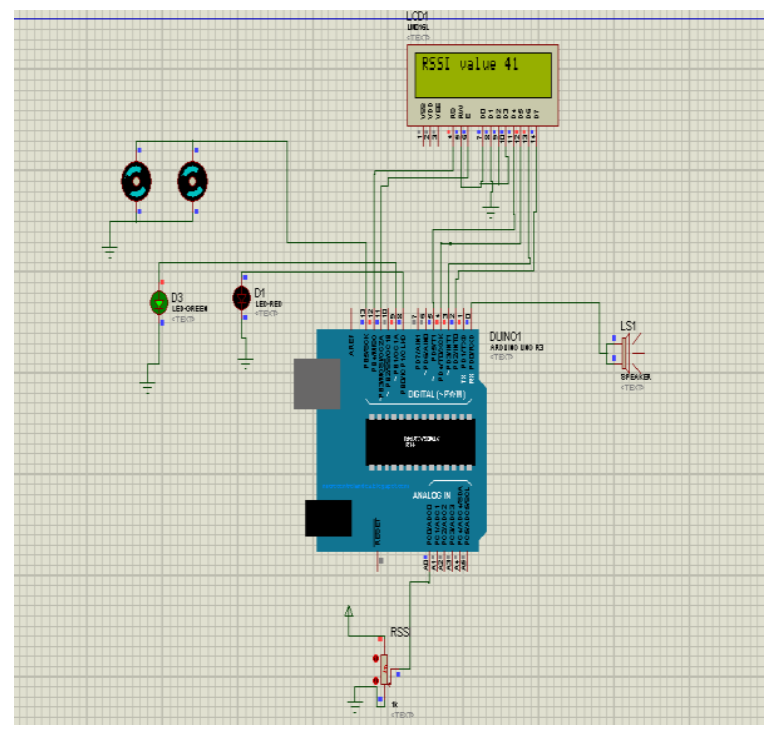

Fig. 5: Simulation Result For Before Arrival Train

In this simulation result in RSSI value below 41 the LCD display show the red signal so, people do not use the mobility platform.

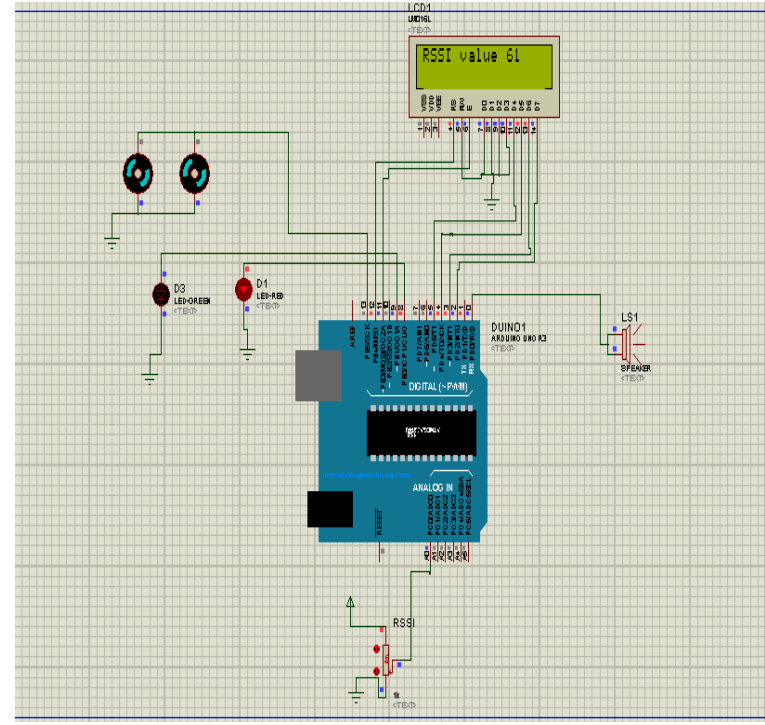

Fig. 6: Simulation Result For After Dispatch Train

In this simulation result in RSSI value above 41 the LCD display show the green signal so, people can move to the platform to another platform.

2)

Hardware Result

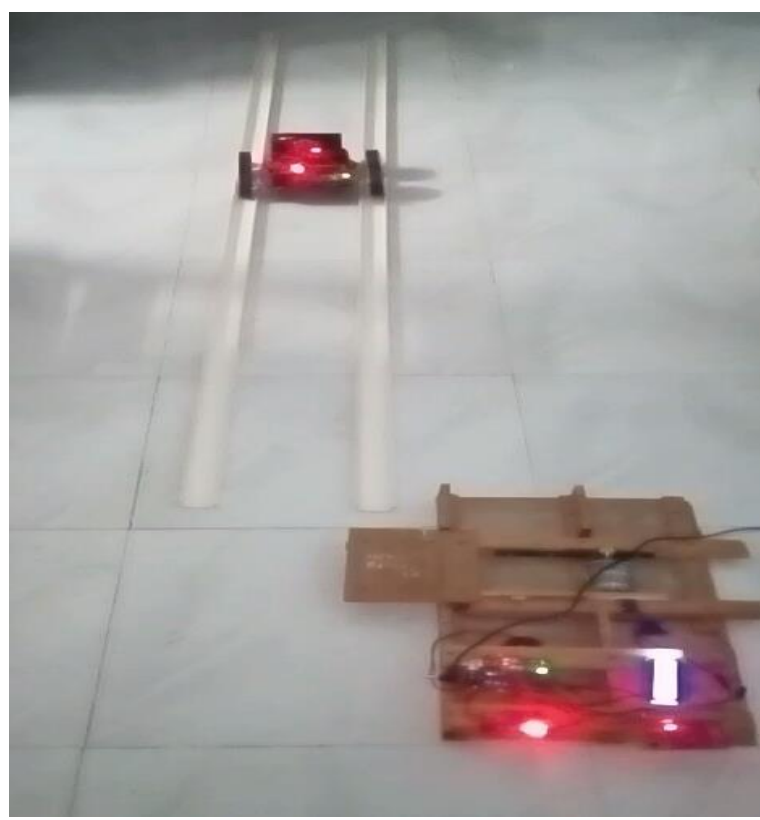

Fig. 7: Hardware Result For Before Arrival Train

In this hardware result mobility platform is open people can use in this platform.

\section{Conclusion}

To developed a mobility platform using embedded system. Each track is to be connected with PIR sensor. Each train is to be connected with RF transceiver, which will send a data of arrival before it reaches the platform. Transceiver and signal obtained matches in frequency then it pass through physical layer and MAC protocol which is interface with micro-controller. Microcontroller instruct to dc motor where dc motor controls the movable rostrum. This project helps old age peoples and psychically challenges peoples to reach the desired platforms. 


\section{Reference}

[1] Adarsh K S, Riya Robert, Kavia E "Railway track pedestrian crossing between two platforms" International Journal of Emerging Technology and Advanced Engineering, (ISSN 2250-2459, ISO 9001:2008 Certified Journal, Volume 5, Issue 12, December 2015).

[2] Sudarsan .P, Ram Kumar. S, Surendar.R, UdaySankar. T, Karthik "Artificial railway platform for domestic railway station" proceedings of 21st IRF International Conference, 15th March 2015, Chennai, India, ISBN: 978-93-82702-78-8

[3] Manu Kanchan \& Ankur Bansal "Conceptual design to transfer handicapped and old people from one railway platform to another" issue on 2014.

[4] G.Prabhavathi, B.Sanjana, S.P.Dhivya"Railway track pedestrian crossing between platforms" IOSR Journal of Electronics and Communication Engineering (IOSRJECE) e-ISSN: 2278-2834, pISSN: 2278- 8735.Volume 9, Issue 2, Ver. III (Mar - Apr. 2014) PP 87-91.

[5] J. Banuchandar, V. Kaliraj, P. Balasubramanian, S. Deepa, N. Thamilarasi "Automated unmanned Railway Level Crossing System", International Journal of Modern Engineering Research (IJMER) www.ijmer.com Vol.2, Issue.1, Jan-Feb 2012 pp-458- 463 ISSN: 2249-6645.

[6] U.S. Department of Transportation, Federal "Highway Administration. Motor Vehicle Accident Costs "(Technical Advisory T7570.2, 31 October)

[7] Silla, A. and J. Luoma (2011). "Effect of Three Counter measures against the Illegal Crossing of Railway Tracks". Accident Analysis and Prevention, Volume 43, Issue 3.

[8] Q.H.Luo,Y.Peng,X.Y.Peng,"Multiple Dimensional Uncertain data stream clustering algorithm," Chin. J. Sci. Instrum. vol. 34, no. 6, pp. 1330-1338, 2013.

[9] A.Vempaty, O. Ozdemir, K. Agrawak, et al, "Localization in Wireless Sensor Networks: Byzantines and Mitigation Techniques," IEEE Trans. on Signal Process., vol. 61, no. 6, pp. 1495-1508, Jun., 2013.

[10] Q. H. Luo, Y. Peng, X. Y. Peng, Abdulmotaleb EI Saddik, "Uncertain Data Clustering-based Distance Estimation in Wireless Sensor Networks," Sensors, vol. 14, no. 4, pp. 6584-6605, April., 2014.

[11] M. F. Snoussi, H. Richard, "Interval-Based Localization using RSSI Comparison in MANETs," IEEE Trans. Aerospace and Electronic Systems., vol. 47, no. 4, pp. 2897-2910, Apr., 2011.

[12] W. L. Zhang, Q. Y. Yin, H. Y. Chen, et al, "Distributed Angle Estimationfor localization in wireless sensor networks," IEEE Transactions on Wireless communications, vol. 12, no. 2, pp. 527 5367, Feb., 2013.

[13] W. Kim, J. Park, J. Yoo, H. J. Kim, C. G. Park, "Target LocalizationUsing Ensemble Support Vector Regression in Wireless SensorNetworks," IEEE Trans. Cybernetics., vol. 43, no. 4, pp. 1189 - 1198, Apr., 2013.

[14] S.V. Manikanthan , T. Padmapriya "An enhanced distributed evolved node-b architecture in 5G tele-communications network" International Journal of Engineering \& Technology (UAE), Vol 7 Issues No (2.8) (2018) 248-254.March2018.

[15] S.V.Manikanthan and T.Padmapriya "Recent Trends In M2m Communications In 4g Networks And Evolution Towards 5g", International Journal of Pure and Applied Mathematics, ISSN NO: 1314-3395, Vol-115, Issue -8, Sep 2017.

[16] T. Padmapriya and V.Saminadan, "Handoff Decision for Multiuser Multiclass Traffic in MIMO-LTE-A Networks", 2nd International Conference on Intelligent Computing, Communication \& Convergence (ICCC-2016) - Elsevier PROCEDIA OF COMPUTER SCIENCE, vol. 92, pp: 410-417, August 2016. 